\title{
Enteric viral infection in human and animal
}

\author{
Yashpal S. Malik · Jelle Matthijnssens
}

Published online: 27 June 2014

(C) Indian Virological Society 2014

Enteric infections leading to acute gastroenteritis rank together with respiratory tract infections as the most common infectious disease syndromes of humans and animals affecting all age groups worldwide. It has been estimated that nearly 5 billion diarrheic episodes occur every year globally and 15-30\% of severely affected patients succumb to it, which is generally higher at the extreme ages of life, particularly in low-income settings of developing countries. The clinical illness caused by enteric pathogens varies but usually involves diarrhea, vomiting and fever, of differing length and severity. Many microbial pathogens are main causes of infectious gastroenteritis and amid them bacterial and parasitic infections have diminished in occurrence as a result of progresses in public health. However, viral gastroenteritis has not dropped in an analogous manner from these interventions.

Enteric viral pathogens recognized since 1970 include viruses mainly belonging to the four families Reoviridae (rotavirus), Caliciviridae (calicivirus), Astroviridae (astrovirus) and Adenoviridae (adenovirus) and are known to cause billions of cases of enteric viral infection severy year. During the last decade, the availability of next-generation sequencing as a new research tool to explore unknown viruses of human and veterinary importance has aided in the detection of putative novel pathogens and the documentation of the etiologic agents of numerous

\footnotetext{
Y. S. Malik $(\bowtie)$

Indian Veterinary Research Institute, Izatnagar 243 122, Uttar Pradesh, India

e-mail: malikyps@yahoo.com

J. Matthijnssens $(\square)$

Laboratory for Clinical and Epidemiological Virology, REGA Institute, Leuven, Belgium

e-mail: jelle.matthijnssens@gmail.com
}

infections, cracking the long-standing anonymities of many genetically diverse and rapidly evolving viruses. This metagenomic approach has been employed in several studies probing fecal samples of livestock and companion animal species, providing evidence on the diversity of the animal fecal virome, helping the elucidation of the etiology of diarrheal disease in animals and identifying potential zoonotic and emerging viruses in humans. In the past few decades, the molecular characterization of many of these gastroenteritis viruses has led to advances both in our understanding of the pathogens themselves as well asin development of state-of-art diagnostics. Although, the use of these highly accurate, reliable and sensitive approaches to identify and characterize individual viral pathogens is in its infancy, it has lightened-up new avenues to review disease burden and contemplate new directions for adopting vaccination, improvements in public health measures and sanitary practices. In particular, the implementation of public health control measures are crucial to reduce disease affliction in developing countries.

With the introduction of novel molecular techniques, many novel viruses have been identified in feces of patients suffering from gastroenteritis including toroviruses, picobirnaviruses, enterovirus and kobuviruses which emphasizes the importance of surveillance. With the exception of a few viruses, the association of the majority of these new viruses with disease causing gastrointestinal infections has not been firmly established. As a result, data on the biology, pathology, as well as epidemiology of these newly identified enteric viruses are still limited. Comprehensive global investigations of the prevalence and diversity of these viruses are required and will be helpful to provide further insight into the pathogenicity, genetic heterogeneity, interspecies transmission potential, and global distribution of these viruses. The probable role of these novel 
viruses as either a 'primary diarrheal agent' in 'immunocompetent hosts'; a 'potential pathogen' in 'immunocompromised individuals' or an 'innocuous virus' in the intestine remains elusive and needs to be investigated despite the reports of the presence of virus in faecal samples of various species of domestic mammals or humans.

Unlike some bacterial pathogens, there are no national notifiable diseases data on the prevalence of enteric viruses. The possible transmissibility of such viruses between animals and humans should be assessed as a number of known and emerging viruses have been associated with enteric infections in animals. These viruses may affect people of all age groups, are often conveyed by fecal contaminated food or water, and thus dependent on control through public health hygienic measures. To better understand and tackle such existing and emerging enteric viral infections there is an urgent need to acquire more scientific knowledge of these pathogens including their reservoir hosts, immunopathobiology, genetic diversity and the development of state-of-art diagnostic tools. Molecular analysis has led to huge advances and whole genome sequence studies of various viruses that are now being regularly published. Against few of the known viral infections vaccines are now being licensed and are inuse. The accumulation of data on pre-and post-vaccine surveillance studies is providing important evidences concerning viral evolution and adaptation. Although progress in the development of improved diagnostic methods are constantly being made, the inaccessibility of preventive and therapeutic measures in various areas of the world is still very worrisome.

This special issue of the Virus Disease journal has a number of articles reviewing rotaviruses, astroviruses, kobuviruses and picobirnaviruses. This issue also includes a review on "exotic rotaviruses" which are most likely derived from various interspecies transmissions coupled with reassortment events, whilst a few other strains exhibit novel genotypes/genetic constellations rarely found in other host species. For example, the surveillance of rotavirus infections in small ruminants (i.e. lambs, goats and camelids) has been a neglected research area in the past. Therefore, a review on "rotaviruses in neglected animal species" was included in the present issue. The prevailing central dogma of rotavirus infection states that the infection is confined in the gastrointestinal tract. However, increasing evidences indicate that rotavirus infections also can be systemic. Experiments on animal models provide pathological and molecular evidences for extra intestinal infection of rotaviruses. To cover this emerging topic a review was invited to highlight the extra-intestinal spread of rotavirus infection. This review has provided data to gain a better understanding of the extraintestinal infections due to rotaviruses.
This special issue on "Enteric Viral infection of Human and Animal" is aimed at providing insights on epidemiology, pathogenesis, diagnosis, prevention, exotic viruses, vaccines, extra-intestinal infections of enteric viral diseases affecting humans and animals. It is hoped that the research and the reviews published in this issue will be a valuable source of scientific information on enteric viral diseases of humans and animals to the scientific community. The Editors of the special issue thanks all the contributors and reviewers for their valuable support.

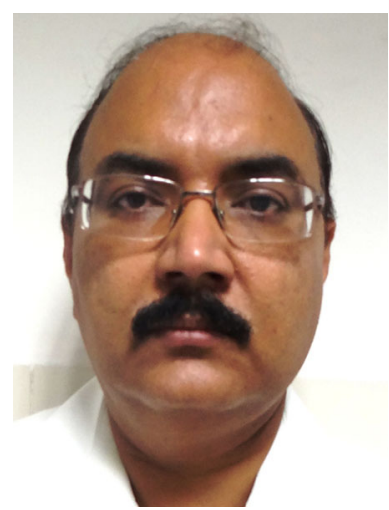

Dr. Yashpal S. Malik is currently working as Senior Scientist at Division of Biological Standardization, Indian Veterinary Research Institute (IVRI), Izatnagar India. He recieved his M.V.Sc and Ph.D. from CCS Haryana Agricultural University, Hisar. He did his postdoctoral research in Molecular Virology at University of Minnesota, Saint Paul, USA. In the past 17 years, he worked in the area of epidemiology, virushost interactions and diagnosis.

Currently, he is working on development of new generation immunodiagnostics using synthetic and recombinant DNA technology. $\mathrm{He}$ is recipient of several awards as recognition of his research achievements. He has published eighty research papers. He has published two books on "Bluetongue" from Indian Council of Agricultural Research, New Delhi and "Trends in Diagnosis of Viral Diseases", published by AgriBioVet Press, New Delhi.

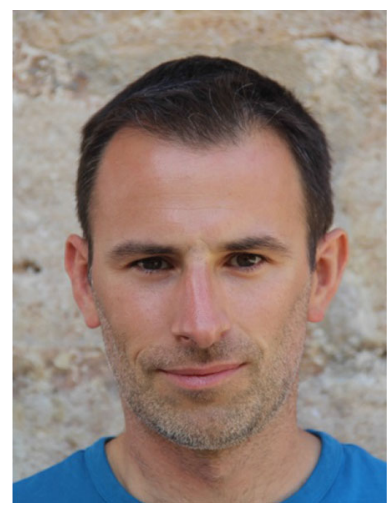

Dr. Ir. Jelle Matthijnssens currently works at the Rega Institute for Medical Research, Laboratory for Clinical and Epidemiological Virology at the University of Leuven in Belgium. He obtained a Ph.D. in Biomedical sciences in 2008 at the University of Leuven. His initial research focuses on the study of complete genomes Dr. Matthijnssens has co-authored more than 85 peer reviewed research papers, and 3 book and genetic diversity of human and animal rotaviruses. More recently, he initiated multiple virome projects investigating the role of the human gut virome in health and disease, and uses viral metagenomics to identify (novel) viruses in undiagnosed clinical samples. Chapters, mainly on gastro-enteritis pathogens and has also been involved in several studies on rotavirus vaccines and clinical trials. Furthermore, Dr. Matthijnssens developed a widely used rotavirus classification and nomenclature system, and is the chair of the Rotavirus Classification Working Group (RCWG). In addition, he is co-responsible for rotavirus matters in the rotavirus study group of the International Committee on Taxonomy of Viruses. 